\title{
Comparing the Communication Mechanisms of Health State Monitoring BGM Devices*
}

\author{
Béla Almási \\ Faculty of Informatics, University of Debrecen \\ almasi.bela@inf .unideb.hu
}

\begin{abstract}
Monitoring the human's health state by continuous or high density parameter measurement is a widely used technology in the medical world. This idea is a standard treatment technology in the diabetes control: The "Self Monitoring of Blood Glucose" (SMBG) technology forces the patient to measure the blood glucose value many times in a day, and precisely log the results into the "diabetes diary" (see [1]). The currently used blood glucose meters (BGMs) are able to store and transmit the measurement data to a computer, so opening the ability of the automation of the monitoring. The different BGM devices usually use their own protocol in the data transmission process. In this paper we study the communication mechanisms of three popular BGM devices: The "Personal Optimum" (produced by the 77 Electronics Ltd; the most popular BGM device in Hungary); the "AccuCheck Active" (Roche's BGM device, widely used in Europe); the "OneTouch Select mini" (produced by LifeScan, and it is a popular BGM device in the USA). We discuss the hardware requirements and introduce a software tool "WinDcont", which can be used to automate the diabetes diary logging for the studied BGMs.
\end{abstract}

Keywords: SMBG, diabetes monitoring, blood glucose meter, communication, data transfer

$M S C: 91 \mathrm{~A} 28,91 \mathrm{E} 45$

\section{Introduction}

Diabetes is a well known common disease in the West-European and North-American countries, about 10 percent of the inhabitants are estimated to be affected by

${ }^{*}$ The research was supported by the TÁMOP-4.2.2.C-11/1/KONV-2012-0001 project. The project has been supported by the European Union, co-financed by the European Social Fund. 
diabetes. Different forecasts tells that this ratio is increasing. Different types of diabetes exist, but a common feature is that the patient's body does not produce enough insulin to keep the blood glucose value at the normal level (below $7 \mathrm{mmol} / \mathrm{l}$ ). Thus the blood glucose value of the patient increases, and special treatments are necessary. One of the modern, currently used diabetes control methods is the so called "Self Monitoring of Blood Glucose" (SMBG) (see [1]-[3]). SMBG means, that the patient does have a blood glucose value meter, and he measures his blood glucose value many times in a day. The patient logs the measurement results into his diabetes diary (see Table 1). The diabetes specialist doctor will check the diary, and (using other laboratory investigations too) decides the necessary treatment steps.

\begin{tabular}{|l|r|r|r|r|r|r|}
\hline Date & $\begin{array}{r}\text { Before } \\
\text { breakfast }\end{array}$ & $\begin{array}{r}\text { After } \\
\text { breakfast }\end{array}$ & $\begin{array}{r}\text { Before } \\
\text { lunch }\end{array}$ & $\begin{array}{r}\text { After } \\
\text { lunch }\end{array}$ & $\begin{array}{r}\text { Before } \\
\text { dinner }\end{array}$ & $\begin{array}{r}\text { After } \\
\text { dinner }\end{array}$ \\
\hline 01.03. & 4,7 & 6,3 & 5,2 & 8,6 & 7,2 & 9,1 \\
\hline 02.03. & 5,8 & 7,2 & 6,7 & & 5,4 & \\
\hline 03.03. & 6,2 & & 7,5 & 9,1 & 6,1 & \\
\hline
\end{tabular}

Table 1: Diabetes diary example

The currently available blood glucose value meters (BGM devices) usually contain memory which may store a few hundreds of measurement results. Another common feature of the modern BGM devices that they offer some kind of computer connection possibility in order to transmit the measurement results from the device's memory to the computer. The communication protocol between the BGM device and the PC is usually vendor-specific: each producer uses his own technology and protocol. In this paper we investigate the communication mechanism of three popular BGM devices: The DCONT Personal Optimum (produced by 77Electronics Ltd, which is a very cheap and very popular device in Hungary), the "AccuCheck Active" (Roche's BGM device, widely used in Europe) and the "OneTouch Select mini" (produced by LifeScan, and it is a popular BGM device in the USA and Worldwide). We discuss the hardware requirements of the PC connectivity, describe the communication mechanisms and introduce a new software tool "WinDcont" (developed at the Faculty of Informatics, University of Debrecen in Hungary), which can be used to automate the diabetes diary logging for the considered BGMs.

\section{DCONT Personal Optimum}

The 77Electronics Ltd produces many different BGM devices ("DCONT Family"). All the devices have an Infrared LED (usually at the top of the device, see Figure 1), which can be used to establish the data transfer to the PC environment. The DCONT devices use a common protocol, so most of the statements in this section are valid also for other BGMs of the 77Electronics. The physical layer protocol of the DCONT family devices is RS-232, which means, that the standard Irda 

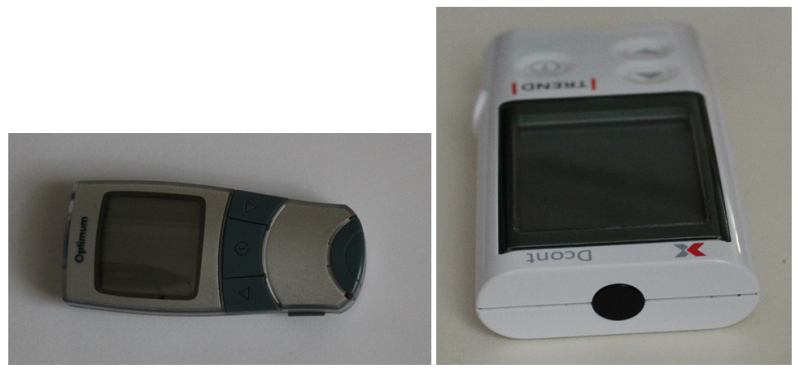

Figure 1: DCONT BGM devices

protocol based infrared ports of the PCs are unusable for connecting the BGM device, special hardware is required. The physical layer signaling uses a simple digital light transmission technology at wavelength of $\lambda=940 \mathrm{~nm}$ (the light pulse means the bit value of zero). For receiving the signals the 77Electronics Ltd offers a receiver hardware (see [4]), but there is a very simple solution based on the Kingbright L-53P3C infrared phototransistor (introduced in [5], see Figure 2).

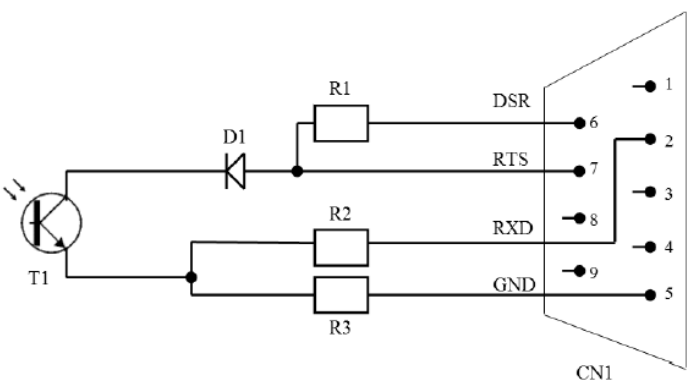

List of components:

$$
\begin{array}{ll}
\text { T1 } & - \text { L-53P3C phototransistor (KINGBRIGHT) } \\
\text { D1 } & -1 N 4148 \text { diode } \\
\text { R2 } & -470 \Omega \text { resistor } \\
\text { R1,R3 } & -2,2 \mathrm{~K} \Omega \text { resistor } \\
\text { CN1 } & \text { - DB9 mother connector (to the PC serial port) }
\end{array}
$$

Figure 2: Infrared receiver circuit for DCONT BGMs

Although the circuit presented in Figure 2 was planned to connect to a serial port (COM port), the L-53P3C phototransistor can be connected to the input port of a CP2102 based USB-TTL UART converter circuit (see Figure 3), thus creating a receiver hardware for USB port with price less than 5 USD (see the prices at http: //stores.ebay.com/ICs-Processors-Store).

The communication mechanism and the record structure of the DCONT receivers is not published by the producer. It uses a simple RS-232 transmission 


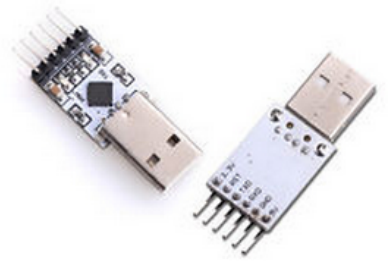

Figure 3: CP2102 USB-TTL UART

with simplex data transfer (i.e. no data is coming from the computer to the BGM device), all the memory content of the device is transmitted to the PC. The RS-232 parameters are "2400-8-N-1" (i.e. 2400 baud, 8 data bit, No parity, 1 stop bit, No flow control). Due to the simplex communication we are not able to transmit only one measurement record (e.g. in the case of data transmission error in one record), we have to transmit the whole memory content.

\section{AccuCheck Active}

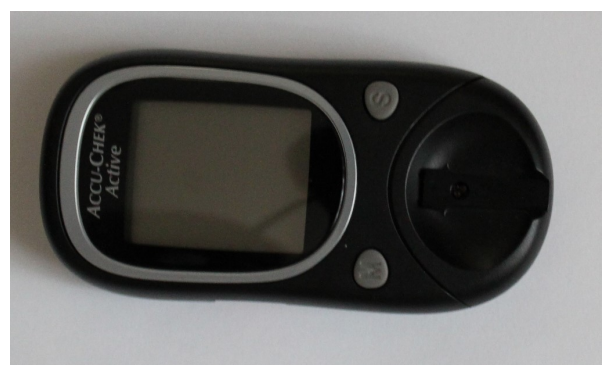

Figure 4: AccuCheck Active Blood Glucose Meter

The AccuCheck Active BGM is a small equipment developed by Roche. It contains an infrared module at the top of the device for communication with the computer. The communication uses RS-232 protocol, so (similarly to the DCONT devices) the standard Irda based PC infrared ports are unusable for connecting the AccuCheck Active BGM to the PC. The physical layer signaling uses a modulated infrared signaling which can be received only by the producer's receiver circuit (see [6]). The RS-232 parameters of the connection are "9600-8-N-1". The communication between the BGM and the PC uses a halfduplex command driven mechanism. The producer (Roche) did not publish the communication protocol (i.e. the commands and the structure of the answers), but S.G Pickering (University of Bath, UK) has decoded and published the communication protocol (see [7]). The command driven mechanism opens the possibility of downloading the measurement 
records one-by-one, so if an error occurs during a record transmission, the record download can be repeated without restarting the whole process.

\section{OneTouch Select Mini}

The Onetouch Mini is a really mini BGM device produced by LifeScan (Johnson\&Johnson). The size is like a half pen, so it is very convenient for travelling. For the computer connection the BGM contains a $3.5 \mathrm{~mm}$ stereo Jack outlet at the right end. The connection is not optical (i.e. not infrared), it uses a wired connection, and so it is much more reliable than the infrared one.

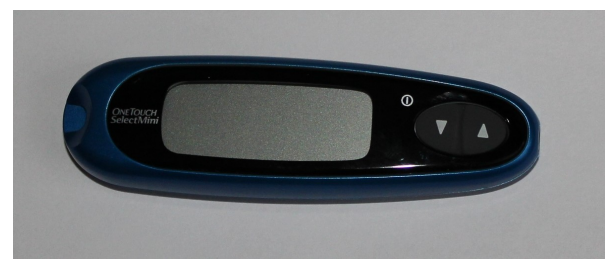

Figure 5: OneTouch Mini BGM device

The physical signaling uses TTL level RS-232 mechanism. For the hardware connection the producer's cable can be used, but the CP2102 based USB-TTL UART converter (see Figure 2) can also be used by connecting a $3.5 \mathrm{~mm}$ stereo Jack plug to the TXD, RXD and GND pins of the converter (so creating a very cheap, less than 5 USD, hardware connection possibility). The RS-232 parameters are "9600-8-N-1" for the OneTouch meter. The communication is a well designed halfduplex command driven mechanism. The commands and the answers are published by the producer (freely available), so it is very easy to develop communication software for the LifeScan BGM. The command driven mechanism opens the possibility to download only one measurement record (so, e.g. if an error occurs in the communication we do not need to repeat the whole download process).

\section{WinDcont Software}

We have created the software routines to implement the communication mechanisms of the discussed BGM devices. The routines are collected in the software named "WinDcont".

The WinDcont software gives a simple and common interface to download the measurement data from the BGM's memory. On the "Settings" screen we can choose the type of the BGM device (see the right side of Figure 6). The data transfer can be started on the main screen (and it will be performed according to the protocol of the selected BGM type). The software is also capable to "merge" the measurement results coming from two (or more) different devices: The 


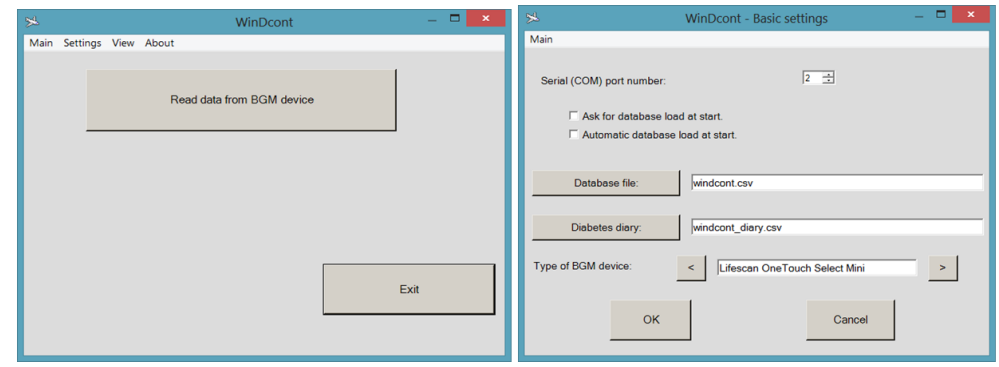

Figure 6: WinDcont software

measurement results will be timely ordered, and the diabetes diary will be created automatically. The WinDcont software can be downloaded from the web: http://irh.inf .unideb.hu/user/almasi/new/index.php/projektek/16

\section{Summary}

In this paper we investigated the communication mechanism of three (very popular) blood glucose meter devices. The vendors use their own mechanism to transmit data from the BGM to the PC, so usually it is not trivial to automate the creation of the diabetes diary when the patient use different BGM devices. The WinDocnt software was introduced in this paper, which can be used to transfer the measurement results from the studied BGM devices. The WinDcont software also offers the possibility of merging the data coming from different meters into one diary.

\section{References}

[1] R. GRAHAM, Self-Monitoring of Blood Glucose (SMBG): Considerations for Intensive Diabetes Management, Pharmacy and Therapeutics, Vol. 20 (2005) Supplement.

[2] E. I. BOUTATI, S. A. RAPTIS, Self-Monitoring of Blood Glucose as Part of the Integral Care of Type 2 Diabetes, Diabetes Care, 32 (2009), 205-210.

[3] U. L. MALANDA, S. D. BOT, G. NIJPELS, Self-Monitoring of Blood Glucose in Noninsulin-Using Type 2 Diabetic Patients, Diabetes Care, Vol. 36 (2013), 176-178.

[4] http://www.e77.hu/webshop/webshop-2/26/262/diabetesz/ P-dcont-hu-enaplo-kezdocsomag (Checked 30.04.2014.)

[5] B. ALMÁSI, Á. MÁRTON, RDCONT - A System for Diabetes Blood Glucose Data Management, 7th International Conference on Applied Informatics, Eger, Hungary (2007), 303-309.

[6] https://www.accu-chek.com/us/data-management/smart-pix.html (Checked 30.04.2014.)

[7] S. G. PICKERING, Decoding the Accu-Chek Active blood glucose meter IR protocol, http://people.bath.ac.uk/enpsgp/Zaurus/accu-chek.html (Checked 30.04.2014.) 